\title{
Hundebisse: bitte melden!
}

Bundesamt für Veterinär-

wesen BVET

Rechtsgrundlage der

Meldepflicht: Tierschutz-

verordnung (in Kraft seit dem

1.9. 2008), Art. 78 Meldung

von Vorfällen

1 Tierärztinnen und Tierärzte, Ärztinnen und Ärzte, Tierheimverantwortliche, Hundeausbilderinnen und Hundeausbilder sowie Zollorgane sind verpflichtet, der zuständigen kantonalen Stelle Vorfälle zu melden, bei denen ein Hund:

a. Menschen oder Tiere erheblich verletzt hat; oder

b. ein übermässiges Aggressionsverhalten zeigt.

2 Die Kantone können die Meldepflicht auf weitere Personenkreise ausdehnen.

Korrespondenz: Regula Kenne

Bundesamt für Veterinärwesen Bereich Kommunikation Schwarzenburgstrasse 155 CH-3003 Bern-Liebefeld Tel. 0313238496 Fax 0313238570

regula.kennel[at]bvet.admin.ch
Hundebisse beim Menschen und bei Tieren müssen seit 2006 gemeldet werden. Wer als Arzt oder Tierärztin eine Bissverletzung behandelt, muss diese dem kantonalen Veterinäramt melden. Die Meldepflicht gilt auch, wenn man als Hundetrainerin oder Tierarzt an einem Hund auffälliges Verhalten beobachtet.

Das System hat sich eingespielt, die Meldepflicht funktioniert im Prinzip. Was sich allerdings verbessern lässt, ist die Meldedisziplin. Noch immer muss von einer Dunkelziffer ausgegangen werden; es werden bei weitem nicht alle Vorfälle gemeldet. Es ist wichtig, dass sich die Ärzte und Tierärzte ihrer Pflicht bewusst sind und wissen, wie im konkreten Fall vorzugehen ist. Als (Tier-)Ärztin und (Tier-)Arzt können Sie einen wichtigen Beitrag leisten zur Prävention von Beissunfällen.

Grundsätzlich müssen Hunde, die ein anderes Tier oder einen Menschen erheblich verletzt haben oder ein übermässiges Aggressionsverhalten zeigen, den kantonalen Veterinärbehörden gemeldet werden. Aber was ist unter «erheblich» genau zu verstehen und was ist gemeint mit «übermässigem Aggressionsverhalten»? Eine Verletzung gilt als erheblich, sobald sie medizinisch versorgt werden muss. Dies gemäss der technischen Weisung des Bundesamtes für Veterinärwesen BVET über Vorfälle mit Hunden. Das heisst, ein Arzt oder eine Tierärztin muss in jedem Fall eine Meldung machen, sobald er/ sie einen Patienten behandelt, der gebissen worden ist. Es macht keinen Unterschied, ob das Opfer ein Mensch ist oder ein anderer Hund. Die Meldepflicht soll präventiv wirken. Es gilt, möglichst früh auf verhaltensauffällige Hunde aufmerksam zu werden. Der Patient oder der Besitzer des verletzten Tieres muss die Angaben seiner Personalien ausdrücklich erlauben. Es ist aber auch möglich, eine Meldung in anonymisierter Form zu machen.

In der Praxis etwas schwieriger anzuwenden ist die Meldepflicht in Bezug auf Hunde, die «ein übermässiges Aggressionsverhalten zeigen». Gemeint sind Verhaltensweisen, die Menschen oder Tiere gefährden, wobei hier ein gewisser Interpretationsspielraum besteht. Man kann davon ausgehen, dass diese Meldepflicht bei der Ärzteschaft, den Hundetrainern und in den Tierheimen gut verankert ist. Aber auch hier liegt die Anzahl der Meldungen wohl weit unter der tatsächlichen Zahl der Vorfälle. Deshalb sollte das Prinzip heissen: lieber einmal zu viel melden als einmal zu wenig.

Eine spezielle Regelung gilt für Tierärztinnen und Tierärzte mit einem Diplom in Verhaltensmedizin. Sie müssen eine Behandlung nicht melden, wenn der Halter/die Halterin von sich aus zur Konsultation kommt oder von einem praktizierenden Tierarzt überwiesen wird. Bedingung ist eine schriftliche Vereinbarung über die nötigen Sicherheitsmassnahmen, und der Halter muss mit den Anweisungen einverstanden sein und diesen auch nachkommen.

\section{Was passiert nach einer Meldung?}

Die zuständige kantonale Stelle muss jeder Meldung nachgehen und den Fall und damit die Gefährlichkeit des Hundes abklären und beurteilen. In rund der Hälfte der gemeldeten Fälle sind keine Massnahmen nötig, aber in jedem dritten Fall wird ein besonderes Risiko festgestellt. Die Verwarnungen, die ausgesprochen werden, betreffen in der Regel die Verletzung der Aufsichtspflicht. Wenn der einzelne Fall schwerwiegt, können weitere Massnahmen angeordnet werden. Sie reichen vom Leinen- oder Maulkorbzwang über die Umplatzierung des Hundes und der Verfügung eines Halteverbots bis zur Tötung des Tieres. Dies als letzte Massnahme, wenn die Sicherheit nicht anders gewährleistet werden kann.

\section{Was man sonst noch wissen muss}

Die Meldeformulare sind bei den kantonalen Veterinärdiensten erhältlich und können auf deren Homepages heruntergeladen werden. Die entsprechenden Links finden sich auf der Homepage des BVET: www.bvet.admin.ch $\rightarrow$ Themen $\rightarrow$ Veterinärdienst Schweiz $\rightarrow$ Kantonale Veterinärdienste. Es ist sicher von Vorteil, ein paar Exemplare des Meldeformulars in der Praxis vorrätig zu haben. Zuständig ist das Veterinäramt desjenigen Kantons, in dem die medizinische Versorgung vorgenommen oder das auffällige Verhalten beobachtet wurde.

\section{Zahlen und Fakten}

2009 sind 5090 Vorfälle mit Hunden gemeldet worden. Die Zahl der Meldungen stieg gegenüber dem Vorjahr um 10 Prozent. Mehr als die Hälfte der Beissunfälle geschieht mit Hunden, die dem Opfer bekannt sind.

Kinder sind besonders gefährdet. Sie werden im Vergleich zu Erwachsenen häufiger gebissen. Bei Kindern sind die Verletzungen zudem schwerwiegender. Mehr als die Hälfte der Bisse betrifft Hals und Kopf, was bei Erwachsenen seltener ist. Ein Viertel der Kinder wird von kleinen Hunden gebissen. Mittelgrosse und grosse Hunde verursachen je etwas mehr als ein Drittel der Bissmeldungen.

Die Angaben zum Hundetyp sind in den Meldungen sehr unterschiedlich. Eine rassebezogene Auswertung der Statistik ist deshalb schwierig. Am häufigsten vertreten sind die Schäfer- und Sennenhunde, also jene Hunde, die in der Schweiz zu den beliebtesten zählen und am häufigsten anzutreffen sind.

(Quelle: Hundebissstatistik 2009. Hrsg. Bundesamt für Veterinärwesen BVET und die Vereinigung der Schweizer Kantonstierärzte und Kantonstierärztinnen.) 